\title{
Factors Associated with the Need for Ventriculoperitoneal Shunting in Patients with Spontaneous Intracerebral Hemorrhage Requiring Emergency Cerebrospinal Fluid Diversion
}

\author{
Ehsan Alimohammadi ${ }^{1} \odot$ Seyed Reza Bagheri ${ }^{1} \quad$ Homa Hadidi ${ }^{2}$ Shabnam Habibi ${ }^{3} \quad$ Akram Amiri $^{2}$
}

Sahar Moradi ${ }^{2} \quad$ Alireza Abdi ${ }^{4}$

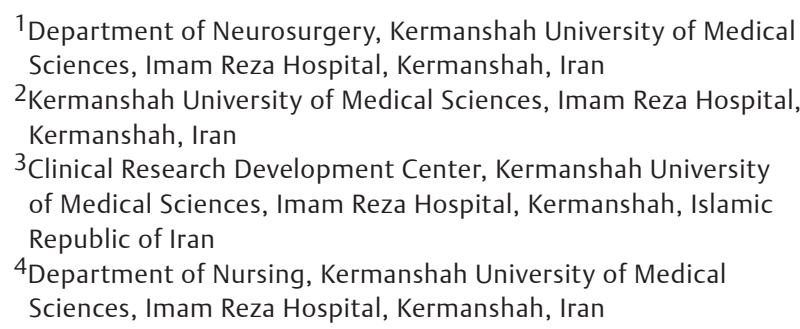

Address for correspondence Ehsan Alimohammadi, MD, Assistant Professor of Neurosurgery, Kermanshah University of Medical Sciences, Imam Reza Hospital, Kermanshah, Iran (e-mail: Hafez125@gmail.com).

Indian J Neurosurg:2020;9:89-94

\begin{abstract}
Keywords

- intracerebral hemorrhage

- hydrocephalus

- cerebrospinal fluid diversion

- external ventricular drain

- ventriculoperitoneal shunt

Introduction Intracerebral hemorrhage (ICH) is a serious medical condition that is frequently complicated by acute hydrocephalus, necessitating emergency cerebrospinal fluid (CSF) diversion in a subset of patients, ultimately requiring long-term treatment via placement of permanent ventricular shunts. The present study aimed to determine factors associated with the need for permanent ventricular shunt placement in these patients.

Methods A total of 309 consecutive patients who underwent emergent CSF diversion with external ventricular drain (EVD) as a treatment for ICH between July 2009 and July 2018 were studied retrospectively to assess the factors that might be correlated with shunt-dependent chronic hydrocephalus. A binary logistic regression model was designed to identify independent related factors of shunt-dependent hydrocephalus after ICH.

Results Of 309 patients included in this study, 102 (33.00\%) required permanent ventricular CSF shunting before discharge. In univariate analysis, age,ventriculitis, ICP elevation $>30 \mathrm{~mm} \mathrm{Hg}$, ICH evacuation, the Graeb score, days of EVD in place, and CSF protein levels were significantly associated with the requirement for permanent CSF diversion $(p<0.05)$. The age and ICH evacuation were protective variables and the ventriculoperitoneal (VP) shunt possibility was reduced by 22.6 and $63.5 \%$, respectively. Conclusion Our results showed that higher Graeb score, ICP elevation $>30 \mathrm{~mm} \mathrm{Hg}$, more days of EVD in place, and higher CSF protein levels were associated with permanent CSF diversion in these patients. Advanced age and ICH evacuation decreased the possibility of VP shunting in our study. These factors may help in predicting which patients will need permanent CSF diversion and could ultimately lead to improvements in the management of these patients.
\end{abstract}




\section{Introduction}

Intracerebral hemorrhage (ICH) is a serious medical condition accounting for 10 to $30 \%$ of all strokes. ${ }^{1,2}$ ICH induces the majority of stroke morbidity and mortality. ${ }^{2}$ Acute onset hydrocephalus is a major complication of ICH, with an incidence of approximately 40 to $50 \%$. Hydrocephalus is an independent predictor of poor clinical outcomes in these patients. ${ }^{3-5}$ In patients with ICH, hydrocephalus mainly develops as a consequence of the intraventricular extension of the hematoma, resulting in intraventricular hemorrhage and impairment of cerebrospinal fluid drainage and reabsorption. ${ }^{6}$ Extensive IVH leads to acute hydrocephalus that compresses the rostral brainstem and thalamus causing damage to the reticular activating system and may reduce cerebral perfusion pressure contributing to ischemic injury. Increased intracranial pressure (ICP) is thought to be fundamental to these injury processes. ${ }^{3,4,7}$ Indeed, patients with considerable IVH, defined as a Graeb score $\geq 6$, have 24 times the odds of developing hydrocephalus, compared with patients who have minimal or no IVH. ${ }^{8}$ Standard treatment of acute hydrocephalus in patients with ICH is emergency CSF diversion via placement of an external ventricular drain (EVD). ${ }^{9}$

Despite intraventricular clot resolution, acute CSF flow obstruction can progress into persistent hydrocephalus and may require the placement of a VP shunt for permanent CSF diversion. ${ }^{10}$

Few studies have been performed about the progression from acute to chronic hydrocephalus and related factors. In this study, we sought to investigate early predictors of long-term shunt dependency in patients with ICH who had received EVD placement for emergency CSF diversion.

\section{Materials and Methods}

We investigated all patients with ICH requiring emergency cerebrospinal fluid diversion admitted to our center between July 2009 and July 2018 retrospectively. The study was approved by the institutional review board of the Kermanshah University of Medical Sciences.

\section{Exclusion}

Patients with ICH secondary to trauma, underlying tumor, or vascular abnormalities were excluded. Patients with age less than 18-years-old or more than 90 -years-old at admission, were excluded too.

Demographic, clinical, and laboratory data, including age, sex, patient admission and discharge status, date of EVD insertion, number of days with an EVD in place, location of EVD, maximum ICP at first, third, and fifth days, and development of ventriculitis, were extracted from the electronic medical records of all patients. Ventriculitis was determined as CSF pleocytosis with low CSF glucose, with or without positive CSF cultures. All CT scans were reviewed by two experienced neurosurgeons.

ICH volume, ICH location, presence of intraventricular hemorrhage (IVH), the IVH volume, presence of third ventricular blood, presence of fourth ventricular blood, temporal horn diameter, and Evan's index were assessed according to the admission brain CT scan of all patients. Evan's index was defined as the ratio of the ventricular width of the bilateral frontal horn to the maximum biparietal diameter. ${ }^{11}$ The Graeb score was calculated from radiographic measurements. ${ }^{12}$ ICH location was classified into sixcategories: cortical, thalamic, basal ganglia, brainstem, cerebellar, and subcortical white matter.

Hydrocephalus was categorized as present or absent, based on temporal horn diameter and Evan's index. We quantified the volume of ICH and IVH using the MIPAV software (version 4.3, National Institutes of Health).

Shunt-dependent hydrocephalus was defined as the presence of clinical symptoms (including symptoms of increased intracranial pressure, decreased mental status, gait disturbance, and/or urinary incontinence) and radiological evidence of ventriculomegaly with Evan's index $>0.3$, necessitating the placement of a permanent VP shunt before hospital discharge.

\section{Statistical Analysis}

Patients were dichotomized into two groups: patients with permanent CSF diversion and those without permanent CSF diversion. Continuous and discrete variables were presented as the mean \pm standard deviation and count with percentage, respectively. Univariate analyses were performed using the Wilcoxon rank sum and the chi-square tests. A binary logistic regression model was constructed with significant variables from the univariate analyses to identify associated factors with VP shunt placement. All statistical analyses were performed using the SPSS software version 20.0 (SPSS Inc., Chicago, IL). The $p$-value $<0.05$ was considered statistically significant.

\section{Results}

A total of 309 consecutive patients with spontaneous ICH and hydrocephalus who underwent emergency cerebrospinal fluid diversion via EVD were included.

The mean patient age was 65.56 (5.96) years. A total of 186 patients (60.2\%) were male. Shunt-dependent hydrocephalus occurred in 102 patients (33.0\%).

Significant differences were found between two groups on the basis of age, ICP elevation $>30 \mathrm{~mm} \mathrm{Hg}$, ICH evacuation, the Graeb score, days of EVD in place, ventriculitis, and CSF protein levels (-Tables $\mathbf{1}$ and $\mathbf{2}$ ).

For predicting the outcome (need for permanent CSF diversion), we used binary logistic regression model, wherein the need for permanent CSF diversion was considered as dependent variable and others, including age, ventriculitis, ICH evacuation, the Graeb score, ICP elevation $>30 \mathrm{~mm} \mathrm{Hg}$, days of EVD in place, and CSF protein levels, were independent variables. The model was fit because the Hosmer-Lemeshow test was not significant $(\mathrm{K} 2=4.84, \mathrm{df}=8$, $p=0.774$ ). The model predicts 66.5 to $92.6 \%$ of the variance of the outcome based on Cox and Snell and Nagelkerke $R$

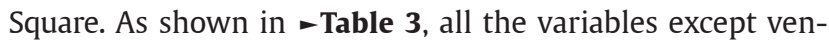
triculitis were associated with the need for VP shunting. 
Table 1 Difference between two groups about qualitative variables

\begin{tabular}{|c|c|c|c|c|}
\hline \multirow{2}{*}{\multicolumn{2}{|c|}{ Variables }} & VP shunt & No VP shunt & Statistical test \\
\hline & & $N(\%)$ & $N(\%)$ & \\
\hline \multirow[t]{2}{*}{ Sex } & Male & $63(33.9)$ & $123(66.1)$ & \multirow{2}{*}{$\begin{array}{l}\mathrm{K} 2=0.157 \\
p=0.692\end{array}$} \\
\hline & Female & $39(31.7)$ & $84(68.3)$ & \\
\hline \multirow[t]{2}{*}{ Ventriculitis } & No & $87(29.8)$ & $205(70.2)$ & \multirow{2}{*}{$\begin{array}{l}\mathrm{K} 2=24.8 \\
p<0.001^{\mathrm{a}}\end{array}$} \\
\hline & Yes & $15(88.2)$ & $2(11.8)$ & \\
\hline \multirow[t]{2}{*}{ Clot in lateral ventricles } & No & $47(34.3)$ & $90(65.7)$ & \multirow{2}{*}{$\begin{array}{l}\mathrm{K} 2=0.187 \\
p=0.665\end{array}$} \\
\hline & Yes & $55(32.0)$ & $117(68.0)$ & \\
\hline \multirow[t]{2}{*}{ Clot in third ventricle } & No & $59(31.9)$ & $126(68.1)$ & \multirow{2}{*}{$\begin{array}{l}\mathrm{K} 2=0.26 \\
p=0.610\end{array}$} \\
\hline & Yes & $43(34.7)$ & $81(65.3)$ & \\
\hline \multirow[t]{2}{*}{ Clot in fourth ventricle } & No & $58(28.4)$ & $146(71.6)$ & \multirow{2}{*}{$\begin{array}{l}\mathrm{K} 2=3.69 \\
p=0.06\end{array}$} \\
\hline & Yes & $44(41.9)$ & $61(58.1)$ & \\
\hline \multirow[t]{2}{*}{ ICP elevation $>30 \mathrm{~mm} \mathrm{Hg}$} & No & $67(60.36)$ & $44(39.63)$ & \multirow{2}{*}{$\begin{array}{l}\mathrm{K} 2=144.67 \\
p<0.001^{\mathrm{a}}\end{array}$} \\
\hline & Yes & $35(17.67)$ & $163(82.32)$ & \\
\hline \multirow[t]{6}{*}{$\mathrm{ICH}$ location } & Cortical & $2(25.0)$ & $6(75.0)$ & \multirow{6}{*}{$\begin{array}{l}\text { K2 (Fisher exact test })=3.9 \\
p=0.066\end{array}$} \\
\hline & Basal ganglia & 43 (31.9) & $92(68.1)$ & \\
\hline & Thalamus & $26(24.3)$ & $81(75.7)$ & \\
\hline & Cerebellar & $24(51.06)$ & $23(48.94)$ & \\
\hline & Subcortical white matter & $3(60.0)$ & $2(40)$ & \\
\hline & Brain stem & $4(57.14)$ & $3(42.85)$ & \\
\hline \multirow[t]{3}{*}{ ICH score } & 3 & $35(27.8)$ & $91(72.2)$ & \multirow{3}{*}{$\begin{array}{l}\mathrm{K} 2=7.41 \\
p=0.061\end{array}$} \\
\hline & 4 & $50(33.1)$ & $101(66.9)$ & \\
\hline & 5 & $17(53.1)$ & $15(46.9)$ & \\
\hline \multirow[t]{2}{*}{ ICH evacuation } & No & $34(21.27)$ & $126(78.73)$ & \multirow{2}{*}{$\begin{array}{l}\mathrm{K} 2=3.39 \\
p=0.028^{\mathrm{a}}\end{array}$} \\
\hline & Yes & $68(52.1)$ & $81(47.9)$ & \\
\hline \multirow[t]{2}{*}{ EVD location } & Frontal & $85(38.8)$ & $134(61.2)$ & \multirow{2}{*}{$\begin{array}{l}K 2=5.56 \\
p=0.061\end{array}$} \\
\hline & Occipital & 17 (18.9) & $73(81.1)$ & \\
\hline \multirow[t]{2}{*}{ GCS category } & $<8$ & $68(37.8)$ & $112(62.2)$ & \multirow{2}{*}{$\begin{array}{l}K 2=3.13 \\
p=0.071\end{array}$} \\
\hline & $\geq 8$ & $34(26.4)$ & $95(73.6)$ & \\
\hline
\end{tabular}

Abbreviations: EVD, external ventricular drain; GCS, glasgow coma scale; ICH, intracerebral hemorrhage; ICP, intracranial pressure; VP, ventriculoperitoneal. aSignificant.

With an increase in the Graeb score, ICP elevation $>30 \mathrm{~mm}$ $\mathrm{Hg}$, and days of EVD in place, the possibility of VP shunting increased 6.091, 7.052, and 16.896 times, respectively. The age and ICH evacuation were protective variables and the possibility of permanent CSF diversion was reduced by 22.6 and $63.5 \%$, respectively ( - Table $\mathbf{3}$ ).

\section{Discussion}

In the present study, of 309 patients with spontaneous ICH who required placement of an EVD due to acute hydrocephalus, 102 patients needed permanent VP shunting. We found age, ICH evacuation, the Graeb score, ICP elevation $>30 \mathrm{~mm} \mathrm{Hg}$, days of EVD in place, and CSF protein levels as independent predictors of shunt-dependent hydrocephalus.

In the study by Zacharia et al, of 210 patients prospectively enrolled in the ICH Outcomes Project, 64 patients required emergency CSF diversion via placement of an EVD. Thirteen of these patients underwent permanent ventricular
CSF shunting before discharge. In univariate analysis, only thalamic hemorrhage $(p=0.008)$ and elevated ICP $(p=0.033)$ were significantly associated with the requirement for permanent CSF diversion. ${ }^{13}$

Miller et al detected that Glasgow Coma Scale score $<9$ $(p=0.033)$, abundant blood in the lateral ventricle $(p=0.016)$, persistent ICP elevations $>20 \mathrm{~mm} \mathrm{Hg}(p=0.017)$, and thalamic ICH location $(p=0.009)$ were associated with VP shunting inunivariate analysis. Thalamic ICH location $(p=0.025)$ and ICP $>25 \mathrm{~mm} \mathrm{Hg}(p=0.025)$ were associated with VP shunting inmultivariate analysis. ${ }^{14}$

In our study, we found that with an increase in the Graeb score the possibility of VP shunting increased 6.091 times.

It remains unclear exactly how IVH contributes to the progression of chronic hydrocephalus although one explanation is that obstruction of the arachnoid villi by blood product, a known factor in the evolution of acute hydrocephalus after IVH, may also contribute to the progression of persistent hydrocephalus. ${ }^{15}$ 
92 Factors Associated with the Need for Ventriculoperitoneal Shunting Alimohammadi et al.

Table 2 Difference between two groups about quantitative variables

\begin{tabular}{|c|c|c|c|c|}
\hline \multicolumn{2}{|l|}{ Variables } & Mean (SD) & Mean rank & Statistical test \\
\hline \multirow[t]{2}{*}{ Age } & VP shunt & $68.41(5.36)$ & 184.04 & \multirow{2}{*}{$\begin{array}{l}Z=8.16 \\
p<0.001^{a}\end{array}$} \\
\hline & No VP shunt & $62.82(5.35)$ & 96.03 & \\
\hline \multirow[t]{2}{*}{ GCS } & VP shunt & $8.05(1.41)$ & 140.13 & \multirow{2}{*}{$\begin{array}{l}Z=2.12 \\
p=0.071\end{array}$} \\
\hline & No VP shunt & $8.34(1.43)$ & 162.33 & \\
\hline \multirow[t]{2}{*}{ ICH score } & VP shunt & $3.82(0.69)$ & 169.73 & \multirow{2}{*}{$\begin{array}{l}Z=2.25 \\
p=0.068\end{array}$} \\
\hline & No VP shunt & $3.63(0.61)$ & 147.74 & \\
\hline \multirow[t]{2}{*}{ Graeb score } & VP shunt & $6.61(1.22)$ & 119.91 & \multirow{2}{*}{$\begin{array}{l}Z=5.06 \\
p<0.001^{a}\end{array}$} \\
\hline & No VP shunt & $7.33(1.14)$ & 172.29 & \\
\hline \multirow[t]{2}{*}{ Evan's index } & VP shunt & $0.347(0.022)$ & 198.09 & \multirow{2}{*}{$\begin{array}{l}Z=1.59 \\
p=0.312\end{array}$} \\
\hline & No VP shunt & $0.313(0.052)$ & 133.77 & \\
\hline \multirow[t]{2}{*}{ ICH volume } & VP shunt & $30.09(13.26)$ & 174.15 & \multirow{2}{*}{$\begin{array}{l}Z=1.64 \\
p=0.421\end{array}$} \\
\hline & No VP shunt & $26.43(8.86)$ & 145.56 & \\
\hline \multirow[t]{2}{*}{ Days of EVD in place } & VP shunt & $8.70(1.45)$ & 251.65 & \multirow{2}{*}{$\begin{array}{l}Z=13.65 \\
p<0.001^{a}\end{array}$} \\
\hline & No VP shunt & $5.57(0.89)$ & 107.37 & \\
\hline \multirow[t]{2}{*}{ ICU stay (D) } & VP shunt & $18.32(2.52)$ & 146.46 & \multirow{2}{*}{$\begin{array}{l}Z=1.18 \\
p=0.236\end{array}$} \\
\hline & No VP shunt & $19.63(6.23)$ & 159.21 & \\
\hline \multirow[t]{2}{*}{ Temporal horn diameter } & VP shunt & $5.91(1.10)$ & 164.66 & \multirow{2}{*}{$\begin{array}{l}Z=1.39 \\
p=0.165\end{array}$} \\
\hline & No VP shunt & $5.78(0.98)$ & 150.24 & \\
\hline \multirow[t]{2}{*}{ Baseline CSF protein (mg/dL) } & VP shunt & $65.31(23.34)$ & 171.23 & \multirow{2}{*}{$\begin{array}{l}Z=1.47 \\
p=0.225\end{array}$} \\
\hline & No VP shunt & $59.31(20.18)$ & 163.65 & \\
\hline \multirow[t]{2}{*}{ CSF protein on day $5(\mathrm{mg} / \mathrm{dL})$} & VP shunt & $55.29(18.98)$ & 196.19 & \multirow{2}{*}{$\begin{array}{l}Z=5.75 \\
p<0.001^{a}\end{array}$} \\
\hline & No VP shunt & $33.95(8.11)$ & 134.70 & \\
\hline \multirow[t]{2}{*}{ Baseline CSF erythrocyte count(cell/mL) } & VP shunt & $147273(18231)$ & 159.81 & \multirow{2}{*}{$\begin{array}{l}Z=1.65 \\
p=0.386\end{array}$} \\
\hline & No VP shunt & $132642(13001)$ & 144.92 & \\
\hline \multirow[t]{2}{*}{ CSF erythrocytes on day 5(cell/mL) } & VP shunt & $41123(11231)$ & 153.21 & \multirow{2}{*}{$\begin{array}{l}Z=1.55 \\
p=0.315\end{array}$} \\
\hline & No VP shunt & $39522(10021)$ & 142.22 & \\
\hline \multirow[t]{2}{*}{ Baseline CSF leukocyte count(cell/mL) } & VP shunt & $821(112)$ & 131.77 & \multirow{2}{*}{$\begin{array}{l}Z=1.73 \\
p=0.446\end{array}$} \\
\hline & No VP shunt & $769(132)$ & 126.65 & \\
\hline \multirow[t]{2}{*}{ CSF leukocyte count on day $5($ cell/mL) } & VP shunt & $1623(231)$ & 162.76 & \multirow{2}{*}{$\begin{array}{l}Z=1.33 \\
p=0.516\end{array}$} \\
\hline & No VP shunt & $1711(276)$ & 168.99 & \\
\hline
\end{tabular}

Abbreviations: CSF, cerebrospinal fluid; EVD, external ventricular drain; GCS, glasgow coma scale; ICH, intracerebral hemorrhage; ICP, intracranial pressure. asignificant.

Table 3 Final binary logistic regression models

\begin{tabular}{|l|l|l|l|l|}
\hline Predictors & B & Sig. & Odds ratio & Cl 95\% \\
\hline Age & -0.256 & $<0.001$ & 0.774 & $0.674-0.889$ \\
\hline Ventriculitis & -2.293 & 0.293 & 1.01 & $0.003-3.55$ \\
\hline Graeb score & 1.80 & 0.021 & 6.091 & $1.311-28.287$ \\
\hline ICH evacuation & -1.008 & 0.012 & 0.365 & $0.167-0.800$ \\
\hline ICP elevation >30 mm Hg & 41.162 & 0.002 & 7.052 & $433138-1.308$ \\
\hline Days of EVD in place & 2.827 & $<0.001$ & 16.896 & $6.008-47.518$ \\
\hline CSF protein level on day 5 & 0.1 & 0.022 & 1.105 & $1.014-1.203$ \\
\hline Constant & -14.283 & 0.016 & $<0.001$ & \\
\hline
\end{tabular}

Abbreviations: CSF, cerebrospinal fluid; EVD, external ventricular drain; GCS, glasgow coma scale; ICH, intracerebral hemorrhage; ICP, intracranial pressure.

ICP elevation $>30 \mathrm{~mm} \mathrm{Hg}$ was also related to an increased risk of shunt-dependent hydrocephalus in our study. Although increased ICP is accepted to be an important predictor of poor outcome following ICH, controlling ICP via medical and surgical treatment may not necessarily improve clinical outcomes or reduce the risk for persistent hydrocephalus. ${ }^{2,4,7}$ 
Ziai et al reported that ICP is not frequently elevated during monitoring and drainage with an EVD in patients with severe IVH. However, ICP $>30 \mathrm{~mm} \mathrm{Hg}$ predicts higher short-term mortality. ${ }^{16}$

Our final logistic model supports a relationship between ICP elevation $>30 \mathrm{~mm} \mathrm{Hg}$ and an increased risk of developing to shunt-dependent hydrocephalus.

Our results showed that $\mathrm{ICH}$ evacuation by surgery reduced the possibility of VP shunting. Other research might point out at the role of blood evacuation on shortening the days of EVD in place, decreasing blood products in CSF circulation, and reducing the ICP may be considered as some explanations for it. ${ }^{5,17,18}$

We found that with an increase in age, the possibility of permanent shunting reduced. One explanation may be the pathophysiological role of age-related cerebral volume loss on decreasing elevated ICP and shunt dependency.

We found an association between high CSF protein levels and risk of permanent shunting in our study. Some studies suggest that high CSF viscosity can lead to an obstructive form of hydrocephalus and/or early CSF circulation disturbances due to arachnoid villi malfunction..$^{1,5,15,19,20}$

\section{Limitations}

Our study has several limitations. This is a retrospective study and some confounding variables may have not been measured and collected owing to the retrospective nature. Findings may be less accurate compared with data from a planned prospective study. Furthermore, the single-center study could have limited the generalizability of our findings. A prospective study in the future may be required to evaluate the actual incidence and predictors of permanent VP shunting in patients with ICH who might require an emergency cerebrospinal fluid diversion. However, in comparison with previous studies, a large sample of patients and the organized group of multiple variant variables are the factors behind the strength of our study.

\section{Conclusion}

Our results showed that higher Graeb score, ICP elevation $>30 \mathrm{~mm} \mathrm{Hg}$, more days of EVD in place, and higher CSF protein level were associated with permanent CSF diversion in these patients. Advanced age and ICH evacuation decreased the possibility of VP shunting in our study.

These factors may help in predicting which patients will need permanent CSF diversion and could ultimately lead to improvements in the management of these patients. .

\section{Note}

All data are available from the corresponding author upon reasonable request.

\section{Authors' Contributions}

E.A., S.R.B.,A.A., and S.H.conceptualized the study and participated in outlining the design. H.H., S.M., and A.A. performed the data acquisition. E.A. and A.A. executed the statistical analysis and wrote the first draft of the manuscript. E.A., S.R.B., H.H., and S.H. revised the final manuscript. All authors have read and approved the manuscript.

\section{Ethics Approval}

The study received ethics approval by the Kermanshah University of Medical Science Ethics Committee.

\section{Funding}

None.

\section{Conflict of Interest}

None declared.

\section{Acknowledgments}

The authors appreciate the Clinical Research Development Center of Imam Reza Hospital for their wise advice.

\section{References}

1 Gaberel T, Magheru C, Parienti JJ, Huttner HB, Vivien D, Emery E. Intraventricular fibrinolysis versus external ventricular drainage alone in intraventricular hemorrhage: a meta-analysis. Stroke 2011;42(10):2776-2781

2 O’Kelly CJ, Kulkarni AV, Austin PC, Urbach D, Wallace MC. Shunt-dependent hydrocephalus after aneurysmal subarachnoid hemorrhage: incidence, predictors, and revision rates. Clinical article. J Neurosurg 2009;111(5):1029-1035

3 Rincon F, Gordon E, Starke RM, et al. Predictors of long-term shunt-dependent hydrocephalus after aneurysmal subarachnoid hemorrhage. Clinical article. J Neurosurg 2010;113(4): 774-780

4 Trifan G, Arshi B, Testai FD. Intraventricular hemorrhage severity as a predictor of outcome in intracerebral hemorrhage. Front Neurol 2019;10:217

5 Yang TC, Chang CH, Liu YT, Chen YL, Tu PH, Chen HC. Predictors of shunt-dependent chronic hydrocephalus after aneurysmal subarachnoid haemorrhage. Eur Neurol 2013;69(5):296-303

6 Zaidi HA, Montoure A, Elhadi A, et al. Long-term functional outcomes and predictors of shunt-dependent hydrocephalus after treatment of ruptured intracranial aneurysms in the BRAT trial: revisiting the clip vs coil debate. Neurosurgery 2015;76(5):608-613, discussion 613-614, quiz 614

7 Li Q, Huang YJ, Zhang G, et al. Intraventricular Hemorrhage and Early Hematoma Expansion in Patients with Intracerebral Hemorrhage. Sci Rep 2015;5:11357

8 Lenski M, Biczok A, Huge V, et al. Role of cerebrospinal fluid markers for predicting shunt-dependent hydrocephalus in patients with subarachnoid hemorrhage and external ventricular drain placement. World Neurosurg 2019;121:e535-e542

9 Klahr AC, Kate M, Kosior J, et al. Early hematoma retraction in intracerebral hemorrhage is uncommon and does not predict outcome. PLoS One 2018;13(10):e0205436

10 Ironside $\mathrm{N}$, Chen CJ, Dreyer V, Christophe B, Buell TJ, Connolly ES. Location-specific differences in hematoma volume predict outcomes in patients with spontaneous intracerebral hemorrhage. Int J Stroke 2020;15(1):90-102

11 Huang YW, Yang MF. Combining investigation of imaging markers (island sign and blend sign) and clinical factors in predicting hematoma expansion of intracerebral hemorrhage in the Basal Ganglia. World Neurosurg 2018;120:e1000-e1010

12 LeRoux PD, Haglund MM, Newell DW, Grady MS, Winn HR. Intraventricular hemorrhage in blunt head trauma: an analysis of 43 cases. Neurosurgery 1992;31(4):678-684, discussion 684-685 
13 Zacharia BE, Vaughan KA, Hickman ZL, et al. Predictors of longterm shunt-dependent hydrocephalus in patients with intracerebral hemorrhage requiring emergency cerebrospinal fluid diversion. Neurosurg Focus 2012;32(4):E5

14 Miller C, Tsivgoulis G, Nakaji P. Predictors of ventriculoperitoneal shunting after spontaneous intraparenchymal hemorrhage. Neurocrit Care 2008;8(2):235-240

15 Garg R, Biller J. Recent advances in spontaneous intracerebral hemorrhage. F1000 Res 2019;8:8

16 Ziai WC, Torbey MT, Naff NJ, et al. Frequency of sustained intracranial pressure elevation during treatment of severe intraventricular hemorrhage. Cerebrovasc Dis 2009;27(4):403-410

17 Wang YM, Lin YJ, Chuang MJ, et al. Predictors and outcomes of shunt-dependent hydrocephalus in patients with aneurysmal sub-arachnoid hemorrhage. BMC Surg 2012;12:12
18 Witsch J, Bruce E, Meyers E, et al. Intraventricular hemorrhage expansion in patients with spontaneous intracerebral hemorrhage. Neurology 2015;84(10):989-994

19 Chen CJ, Ding D, Ironside N, et al; ERICH investigators. Predictors of surgical intervention in patients with spontaneous intracerebral hemorrhage. World Neurosurg 2019;123:e700-e708

20 Paisan GM, Ding D, Starke RM, Crowley RW, Liu KC. Shuntdependent hydrocephalus after aneurysmal subarachnoid hemorrhage: predictors and long-term functional outcomes. Neurosurgery 2018;83(3):393-402 